\title{
Caracterización de la velocidad y dirección de viento en la provincia de Chimborazo
}

\section{Characterization of speed and wind direction in the province of Chimborazo}

Diana Katherine Campoverde Santos. ${ }^{1}$, Amalia Isabel Escudero Villa. ${ }^{2}$, \& Silvia Mariana Haro Rivera. $^{3}$

Abstract.

DOI: https://doi.org/10.33262/concienciadigital.v3i1.1.1156

The present research main shows the behavior of speed and direction wind in Chimborazo province; data were taken from 2014 to 2017, it was applied in eleven weather stations located in strategic places inside study area. Also, database was structured according to the WorPlot software requirements for elaboration of wind roses. A descriptive statistical analysis was performed to characterize their behavior, contrast of hypothesis was carried out to identify significant differences on a monthly, annual and stations. Similar behaviors were determined in the wind data between the Tunshi and Urbina stations with respect to the wind direction in months each year; Alao, Cumandá, Espoch, Matus, Multitud, Quimiag, San Juan and Tunshi stations have wind speeds that reached $2.0 \mathrm{~m} / \mathrm{s}$, while in Atillo, Tixán and Urbina around $3.0 \mathrm{~m} / \mathrm{s}$ at $4.5 \mathrm{~m} / \mathrm{s}$, determining that the areas with the highest availability of wind energy are located in Tixán and Urbina with speeds exceeding $4 \mathrm{~m} / \mathrm{s}$. Finally, with the help of Google Earth, the wind roses were visualized in each station in order to show predominant characteristics through variables studied.

Keywords: Statistical Analysis, Wind Speed, Wind Direction, Chimborazo Province.

\section{Resumen.}

La presente investigación muestra el comportamiento de la velocidad y dirección de viento en la provincia de Chimborazo, se tomaron los datos del 2014 al 2017 de 11 estaciones meteorológicas

\footnotetext{
${ }^{1}$ Escuela Superior Politécnica de Chimborazo, Facultad de Ciencias Pecuarias. Riobamba, Ecuador, diana.campoverde@espoch.edu.ec

${ }^{2}$ Escuela Superior Politécnica de Chimborazo, Facultad de Ciencias. Riobamba, Ecuador., aescudero@espoch.edu.ec

${ }^{3}$ Escuela Superior Politécnica de Chimborazo, Facultad de Ciencias. Riobamba, Ecuador., sharo@espoch.edu.ec
} 
ubicadas en lugares estratégicos de la zona de estudio. Se estructuró una base de datos de acuerdo con los requerimientos del software WorPlot para la elaboración de las rosas de viento. Se realizó un análisis estadístico descriptivo para caracterizar su comportamiento, se efectuaron contrastes de hipótesis para identificar diferencias significativas en forma mensual, anual y por estaciones. Se determinaron comportamientos similares en los datos de viento entre las estaciones Tunshi y Urbina respecto a la dirección de viento en todos los meses de cada año año; las estaciones Alao, Cumandá, Espoch, Matus, Multitud, Quimiag, San Juan y Tunshi presentan velocidades de viento que alcanzaron los $2.0 \mathrm{~m} / \mathrm{s}$, mientras que en Atillo, Tixán y Urbina de $3.0 \mathrm{~m} / \mathrm{s}$ a $4.5 \mathrm{~m} / \mathrm{s}$, determinándose que las zonas con mayor disponibilidad de energía del viento se encuentra en Tixán y Urbina con velocidades que superan los $4 \mathrm{~m} / \mathrm{s}$. Finalmente con la ayuda de Google Earth se visualizó las rosas de viento en cada una de las 11 estaciones con el fin de mostrar características predominantes de las variables estudiadas.

Palabras claves: Análisis estadístico, Velocidad de viento, Dirección de Viento, Provincia Chimborazo

\section{Introducción.}

El estudio estadístico de variables meteorológicas es de vital importancia, puesto que permite identificar características relevantes de su comportamiento, a la vez que son útiles y coadyuvan a estudios de diversas áreas como por ejemplo: el análisis de la relación entre temperatura y precipitación con Aedes aegypti (Bultó et al., 2008) que es el principal vector del dengue común y hemorrágico (Cruz, Sebrango, Hernández, Pina, Marquetti y Sánchez, 2010); la variabilidad del cambio climático y su impacto en la salud humana (Ortíz, Pérez, Rivero, Pérez, Cangas y Lecha, 2008), la temperatura del aire, humedad relativa, velocidad del viento, radiación solar y precipitaciones para la estimación de la evapotranspiración de tomate usando el modelo interacción Suelo (Olioso, Ortega, Valdés y Antonioletti, 2005); la temperatura, humedad relativa, nubosidad, dirección y velocidad del viento para modelar la predicción de heladas mediante redes neuronales (Ovando, Bocco y Sayago, 2005); la descripción de la variabilidad del viento, nivel del mar y temperatura en la costa norte de Chile (Pizarro, Hormazabal, González y Yañez, 1994); frecuencia e intensidad de viento en Zaragoza de las medias mensuales según las direcciones de las rosas de los vientos (Navarro, 1990), el análisis de estacional característico en las turbinas en Manisa eólicas así como el potencial energético eólico, (Tamura, 2015), entre otros.

La energía eólica se produce como resultado de la transformación de la energía cinética del aire en movimiento, siendo aprovechada mediante el uso de dispositivos y/o aerogeneradores que operan de forma aislada o en grupos (parques eólicos) convirtiéndola en energía de diversos tipos, siendo la más común la energía eléctrica (De la Cruz Buelvas, Valencia y Vanegas, 2018; Quiñonez, Huanca y Holguino, 2019).

En los últimos años a nivel mundial existe interés en buscar nuevas fuentes de energía que no 
generen contaminación en el medio ambiente (reduciendo efectos nocivos) con fuentes renovables (energía eólica y solar) que no requieran el uso de reservas de petróleo y gas (Carta, Ramírez y Velázquez, 2009; Watson, 2014). Köse (2004) afirma que: dado que la energía eólica es un recurso local y de medio ambiente limpio y ecológico, es vital llevar a cabo la viabilidad técnica y económica requerida, por lo que debería intensificarse investigaciones que viabilicen el uso de este recurso que coadyuvará a la superación los problemas actuales de energía (p. 1632) especialmente en aquellos que surgen por el difícil acceso, pero que sin embargo están habitadas por familias agricultoras.

Siendo la velocidad y dirección de viento un recurso limpio y renovable del que se puede aprovechar para cubrir necesidades de la sociedad en general, en la última década, numerosos estudios relacionados con la caracterización del viento y potencial de energía eólica se han realizado en diferentes países (Gökçek et al.,2007; B. Natalini y M. Natalini, 2013), la expresión utilizada en estos estudios de energía eólica para representar las distribuciones de viento es la distribución de Weibull.

$P(v)=\left(\frac{k}{c}\right)\left(\frac{V}{c}\right)^{k-1} \cdot e\left(-\frac{V}{c}\right)^{k}$, donde $P(v)$ : es la probabilidad de ocurrencia de una velocidad de viento determinada, $c$ : factor de escala y $k$ : es el factor de forma (Cellura M., Cirrincioneb G., et al, 2008; Ciampoli, Petrini, y Augusti, 2011); dichos estudios han detectado que la velocidad de los vientos disminuye considerablemente durante los meses de invierno, estas variaciones son causadas por las diferencias de temperatura existentes por la desigualdad del calentamiento en las diversas zonas de la tierra (Cúneo, Cerne y Llano, 2018; Hennessey, 1977).

Sin embargo los resultados no se pueden generalizar para todos los países del mundo debido a sus características geológicas propias, es por ello que en Ecuador se viene incentivando el estudio de variables meteorológicas con el fin de aprovechar estos recursos naturales y con ello a aportar con el cuidar el medio ambiente, por ello se han instalado estaciones meteorológicas en diferentes provincias del país, entre estas en la provincia de Chimborazo, la cual se encuentra a una altura de 156 m.s.n.m. hasta los 6310 m.s.n.m.

Los asentamientos poblacionales están entre 2200 a 3300 m.s.n.m., según el Instituto Geográfico Militar (IGM) cuenta con una extensión territorial cercana a los $6500 \mathrm{~km}^{2}$, tiene fértiles valles como los de Guano, Riobamba y Penipe. La provincia tiene importantes afluentes; entre los principales están: el Chambo, Chanchán y Chimbo. Debido a la presencia de masas de aire amazónicas y oceánicas; y sobre todo la altura, se presentan cuatro tipos de climas; a los 4.600 ms.n.m. es de tipo glacial, entre los 3.000 y 4.000 ms.n.m. es páramo, bajando; a los 2.000 ms.n.m. el clima es mezotérmico seco; y en zonas cercanas a la costa el clima es mesotérmico húmedo y semi-húmedo (Toainga, 2015), por esta presencia de masas se da la necesidad de analizar el comportamiento metereológico del viento. 
En Chimborazo, un estudio realizado por Haro, Lema, Meneses y Escudero (2020), mediante el análisis de componentes principales de velocidad de viento en el año 2015, enumeran dos tipos de comportamientos; el primero corresponde a la región norte de la provincia y el segundo a la región del sub trópico, la primera región se caracteriza por poseer zonas ubicadas a una altura superior que los 1.000 ms.n.m. totalmente diferente a la segunda que tiene mayor diversidad con fauna y flora. Estos resultados son útiles para identificar zonas con velocidades de viento con posibilidades de producir energía eólica.

La velocidad de viento está estrechamente asociada a su dirección, es por ello que en el presente trabajo de investigación se muestra un análisis estadístico exploratorio sobre la velocidad y dirección del viento, representando su comportamiento mediante rosas de viento graficadas en worplot usando google earth se las ubicó en cada zona geográfica de cada estación meteorológica que conforma la red de estaciones del Instituto Nacional de Eficiencia Energética y Energías Renovables y la Escuela Superior Politécnica de Chimborazo (INER - ESPOCH), con el fin de identificar patrones de comportamientos donde las variables en estudio presenten características similares.

La red está conformada por 11 estaciones meteorológicas, ubicadas de manera estratégica en toda la provincia (Tabla 1 y Figura 1).

Tabla 1: Ubicación geográfica de las estaciones meteorológicas de la red INER-ESPOCH.

\begin{tabular}{ccccc}
\multirow{2}{*}{ No. } & \multirow{2}{*}{ Nombre de la estación } & \multicolumn{2}{c}{ Coordenadas (UTM) } & Altura \\
\cline { 3 - 4 } & & Longitud & Latitud & (m.s.n.m) \\
\hline 1 & Alao & $773499 \mathrm{E}$ & $9793173 \mathrm{~S}$ & 3064 \\
2 & Atillo & $772610 \mathrm{E}$ & $9758048 \mathrm{~S}$ & 3467 \\
3 & Cumandá & $706262 \mathrm{E}$ & $9755580 \mathrm{~S}$ & 331 \\
4 & ESPOCH & $758398 \mathrm{E}$ & $9816965 \mathrm{~S}$ & 2754 \\
5 & Matus & $777402 \mathrm{E}$ & $9827879 \mathrm{~S}$ & 2685 \\
6 & Multitud & $722699 \mathrm{E}$ & $9764910 \mathrm{~S}$ & 1483 \\
7 & Quimiag & $770084 \mathrm{E}$ & $9816393 \mathrm{~S}$ & 2709 \\
8 & San Juan & $746596 \mathrm{E}$ & $9818849 \mathrm{~S}$ & 3232 \\
9 & Tixán & $747159 \mathrm{E}$ & $9757355 \mathrm{~S}$ & 4240 \\
10 & Tunshi & $764087 \mathrm{E}$ & $9806678 \mathrm{~S}$ & 2840 \\
11 & Urbina & $754533 \mathrm{E}$ & $9835326 \mathrm{~S}$ & 3642
\end{tabular}

Fuente: INER-ESPOCH-UNACH.

Elaborado por: Ramos, C., Pérez, N. 
Figura 1. Ubicación geográfica estaciones meteorológicas de la red INER-ESPOCH

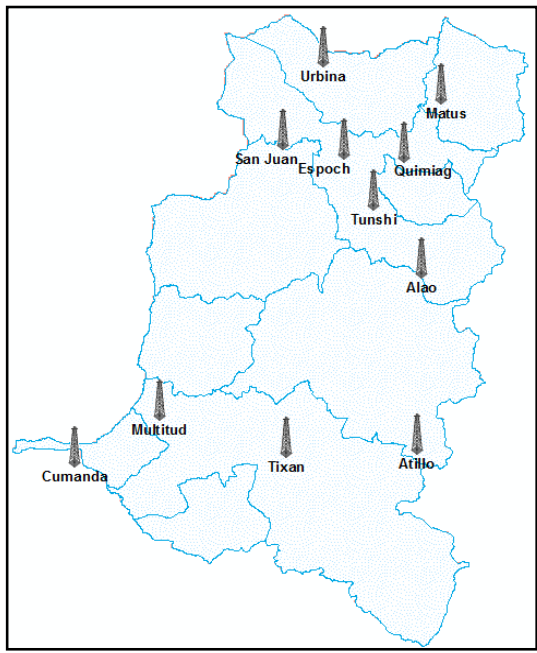

\section{Fuente: Datos INER-ESPOCH-UNACH}

Elaborado por: Ramos, C., Pérez, N.

Se tomaron las velocidades de viento por hora en $\mathrm{m} / \mathrm{s}$ y sus direcciones en grados registrados del 2014 al 2017 mediante el anemómetro con calibración del 5\% y almacenados en Data Logger NRG Symphonie, Los datos se analizaron de forma mensual, anual y por estaciones, de los cuales se hallaron velocidades medias y máximas, con el fin de identificar diferencias significativas entre el comportamiento de dichas variables con respecto al mes, año y estación, para lo cual se usó el contraste de hipótesis t-student y para probar su normalidad el test de Shapiro Wilks, posterior a este análisis se utilizó el software libre Worplot especialista en la construcción gráfica de rosa de vientos para la representación gráfica de los datos de viento de cada una de las estaciones en estudio.

Este estudio fue realizado con el fin de identificar patrones de comportamiento de las variables estudiadas, ya sean en forma mensual, anual o por estaciones, sin embargo, se ha logrado identificar la existencia de microclimas debido a la estructura geográfica de la zona estudiada. Los resultados obtenidos servirán de punto de partida para realizar análisis estadísticos con técnicas avanzadas como: análisis estadístico de datos funcionales, redes neuronales, modelación espacial funcional para estimar datos en zonas no muestreadas, imputación de datos metereológicos faltantes, entre otros, tanto de las variables velocidad y dirección de viento como de otras variable meteorológicas (Rivera y Escudero, 2017; Tamura, 2015), con el fin de conocer a profundidad el comportamiento de dichas variables en la provincia de Chimborazo.

\section{Metodología}

La transmisión de la información de las estaciones meteorológicas se la realiza automáticamente mediante el servicio general de paquetes vía radio (General Packet Radio Service) (GPRS) en los lugares en donde existe cobertura de la red celular, y mediante un modem GOES en donde no existe dicha cobertura. En la figura 5 se explica el proceso de transmisión de la información hasta 
el servidor Protocolo de transferencia de archivos (en inglés File Transfer Protocol o FTP) FTP en la ESPOCH. Para la transmisión GOES se realizaron gestiones, con el objetivo de obtener un canal de transmisión por parte por parte del Instituto Nacional de Meteorología e Hidrología (INAMHI). En el estudio se emplearon datos por hora, información recolectada del Data Logger y mediante una hoja de Excel, se estructuró una base de datos de acuerdo con los requerimientos del análisis, considerando las variables de velocidad y dirección de viento. La detección de atípicos se hizo mediante el método de direcciones ortogonales de máxima y mínima curtosis de forma horaria por mes, este procedimiento consiste en eliminar provisionalmente los datos extremos (sospechosos) en estas direcciones ortogonales, luego calcular la media y la matriz de covarianza con los datos no sospechosos y después identificar los datos atípicos (valores mayores que $\mathrm{p}+3 \sqrt{2} \mathrm{p}$ ) como aquellos que son extremos con la distancia de Mahalanobis: $d_{R}^{2}\left(\mathrm{x}_{\mathrm{i}}, \bar{x}_{R}\right)=\left(x_{i}-\bar{x}_{R}\right) S_{R}^{-1}\left(x_{i}-\bar{x}_{R}\right)^{\prime}$ calculada con las estimaciones sin datos atípicos, este procedimiento se lo realizó usando el algoritmo "Mskurt" del paquete "Outliert" de Matlab (Peña, 2013)

Para la elaboración de las rosas de viento se dividieron los 360 grados en 12 sectores de 30 grados, formando un grupo de cuatro direcciones primarias y ocho secundarias que son: Norte, Este, Sur, Oeste, Nor-noreste, Este-noreste, Este-sureste, Sur-sureste, Sur-suroeste, Oeste-suroeste, Oestenoroeste, Nor-Noroeste. Estas direcciones están representadas por números, los cuales varían de acuerdo con las manecillas del reloj, iniciando con $360^{\circ}$ en el norte, teniendo el este con $90^{\circ}$, el sur con $180^{\circ}$ y el oeste con $270^{\circ}$. Debido a las características propias de la localidad se dividió los datos de velocidad de acuerdo con su magnitud en 5 intervalos: de 0-3, 3-5, 5-7, 7-9 y de 9 en adelante, los registros de 0 son reportados como calma (Bichet, Wild, Folini, y Schär, 2012). Una vez obtenidas las distribuciones de frecuencias como tablas de contingencia en Excel de las dos variables clasificadas, se importaron al Worplot View para construir las rosas de viento, que son gráficas de las dos variables en coordenadas polares con barras que indican el porcentaje de velocidad de viento alcanzado y direcciones predominantes en cada intervalo. Debido a que se visualizó similitud en direcciones de viento entre las estaciones de Tunshi y Urbina se utilizó R para realizar un contraste de hipótesis t-student para identificar diferencias significativas entre dichas estaciones ya que las dos variables siguen distribución normal según el contraste de normalidad de Shapiro. Finalmente se elaboraron mapas temáticos de la provincia de Chimborazo según la ubicación geográfica de cada una de las estaciones meteorológicas, para facilitar la visualización del comportamiento de las dos variables estudiadas.

Worplot View genera automáticamente una lista de horas donde existe faltantes, misma que aparece haciendo clic en la opción Incomplete/Missing Hours de la pestaña Met Data Information, mismos que son imputados automáticamente por el software, mediante el promedio de las cuatro horas que rodean el dato faltante, a excepción de las dos primeras y dos últimas horas, si faltan más de dos horas consecutivas toma los promedios de 5 o más datos a cada lado del vacío (Atkinson y Russell, 1992). 


\section{Resultados}

Variaciones anuales: En los análisis estadísticos se obtuvieron las velocidades medias anuales (Tabla 2), se halló que durante los cuatro años de estudio el comportamiento es similar, verificando mayor variabilidad aproximadamente de una unidad en las estaciones de Alao, San Juan, Tixán y Urbina. En el año 2015 se identificó mayor velocidad de viento promedio en las estaciones de Alao, Atillo, Espoch, San Juan, Tixán y Urbina en los 4 años de estudio, mientras que, en el 2017, la menor velocidad en 8 de las 11 estaciones meteorológicas que son: Alao, Cumandá, Espoch, Matus, Quimiag, San Juan, Tixán y Urbina (Figura 2). La velocidad de viento incrementa en función a la altura geográfica del lugar donde están instaladas las estaciones. Para complementar el estudio se realizó un contraste de hipótesis t-student una vez comprobado que los datos siguen distribución normal mediante la prueba de Shapiro Wilks, se mostró que no hay diferencias significativas entre las velocidades de viento por año de dicha estación, al 95\% de confiabilidad. En cuanto a las demás estaciones todas tienen diferencias significativas con un valor de p igual a 0,000 con un nivel de significancia de 0,05 .

Tabla 2: Promedios anuales de velocidad de viento de las 11 estaciones meteorológicas de la Provincia de Chimborazo.

\begin{tabular}{cccccc}
\hline $\mathbf{N}^{\circ}$ & Año & $\mathbf{2 0 1 4}$ & $\mathbf{2 0 1 5}$ & $\mathbf{2 0 1 6}$ & $\mathbf{2 0 1 7}$ \\
\hline 1 & Alao & 1,93 & 2,01 & 2,01 & 1,71 \\
2 & Atillo & 3,06 & 3,7 & 3,37 & 3,22 \\
3 & Cumandá & 0,74 & 0,6 & 0,48 & 0,36 \\
4 & Espoch & 1,89 & 2,13 & 1,98 & 1,82 \\
5 & Matus & - & 1,52 & 1,68 & 1,36 \\
6 & Multitud & 0,42 & 0,39 & 0,2 & 0,21 \\
7 & Quimiag & 1,84 & 1,93 & 1,95 & 1,69 \\
8 & San Juan & 1,73 & 2,31 & 2,13 & 1,53 \\
9 & Tixán & 3,59 & 4,66 & 4,24 & 3,41 \\
10 & Tunshi & 1,4 & 1,46 & 1,47 & 1,65 \\
11 & Urbina & 3,76 & 4,53 & 4,11 & 3,53 \\
\hline
\end{tabular}

Elaborado por: Diana Campoverde

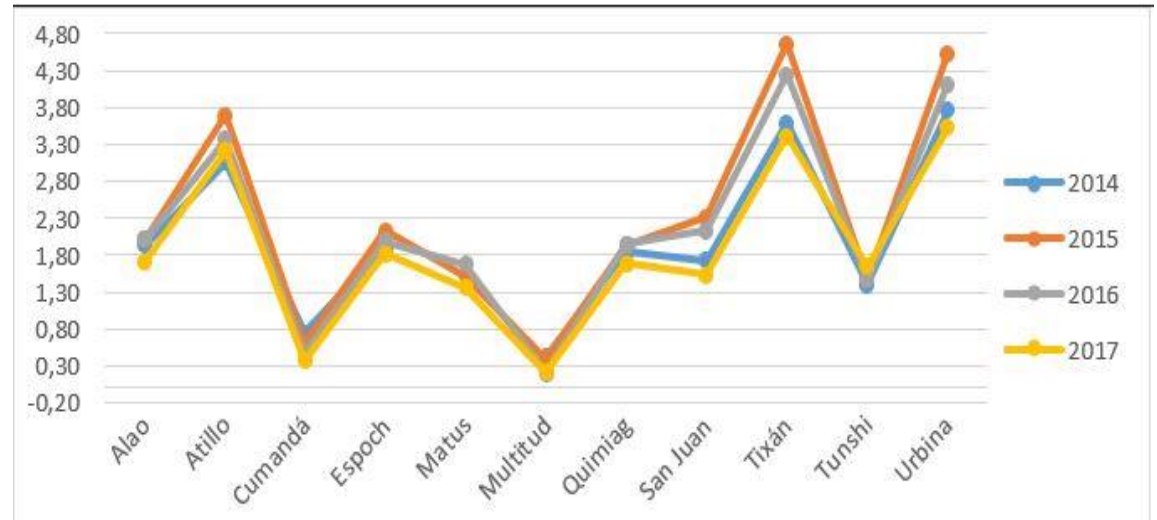

Figura 2. Velocidades de viento medios por estación meteorológica Elaborado por: Diana Campoverde 
Para identificar el comportamiento de las direcciones de viento predominantes se construyeron gráficos apilados (Figura 3). Multitud, Tunshi y Urbina muestran direcciones de viento predominantes hacia el $\mathrm{N}$ durante el 2014; por otro lado, Atillo, Cumandá, Espoch, Multitud y Tixán con direcciones de viento predominantes hacia el E; mientras que las estaciones Alao, Espoch y San Juan presentan características de vientos predominantes hacia el ESE. Cumandá presenta un viento predominante hacia el OSO y Quimiag hacia el NE (Figura 3 (a)).

Figura 3. Direcciones predominantes de velocidad de viento, (a) 2014, (b) 2015, (c) 2016, y (d) 2017.
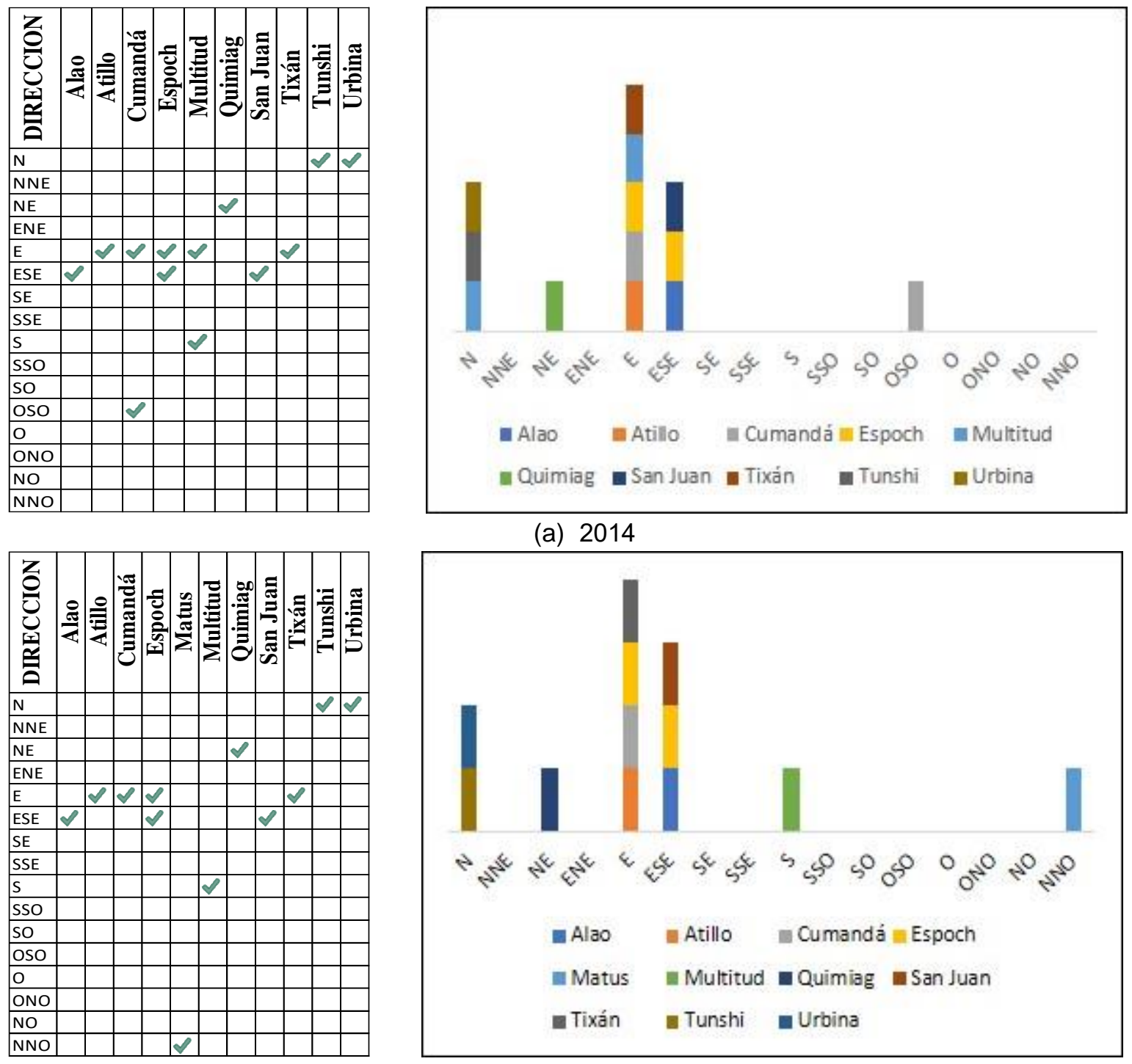

(a) 2014

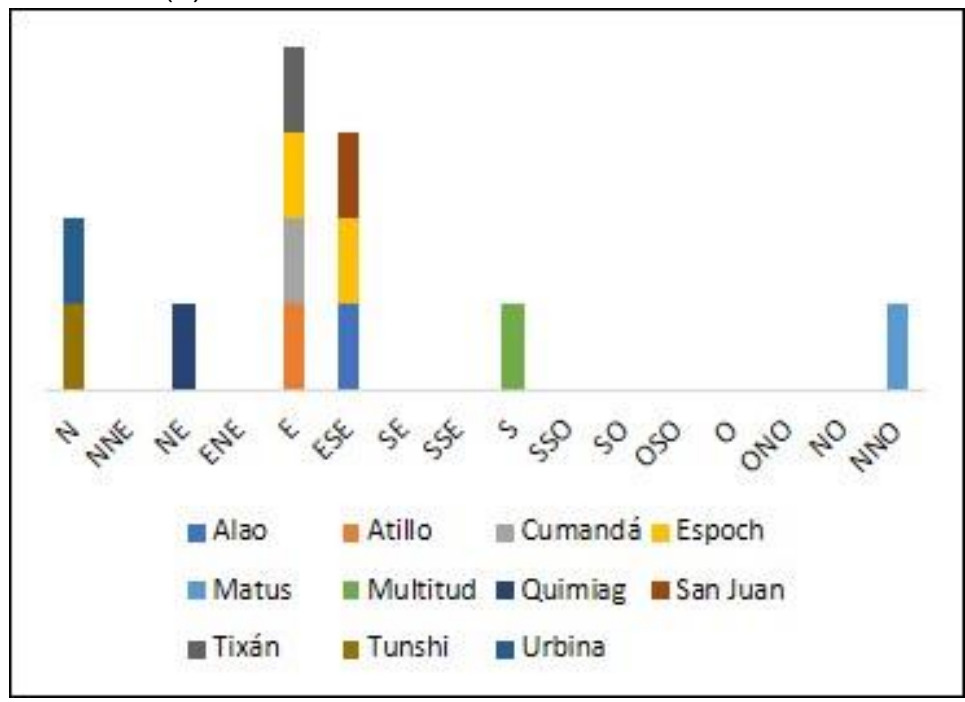

(b) 2015 

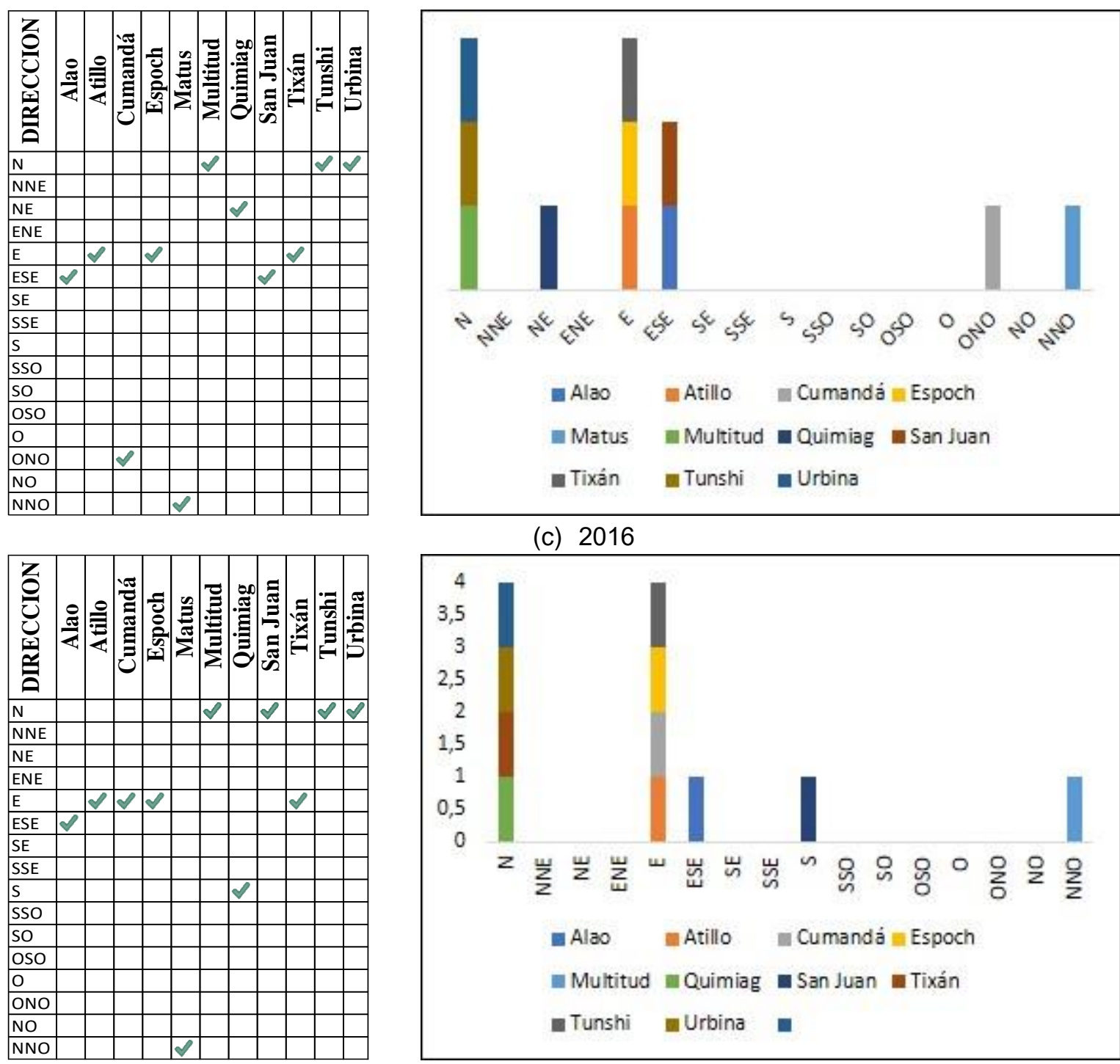

(c) 2016

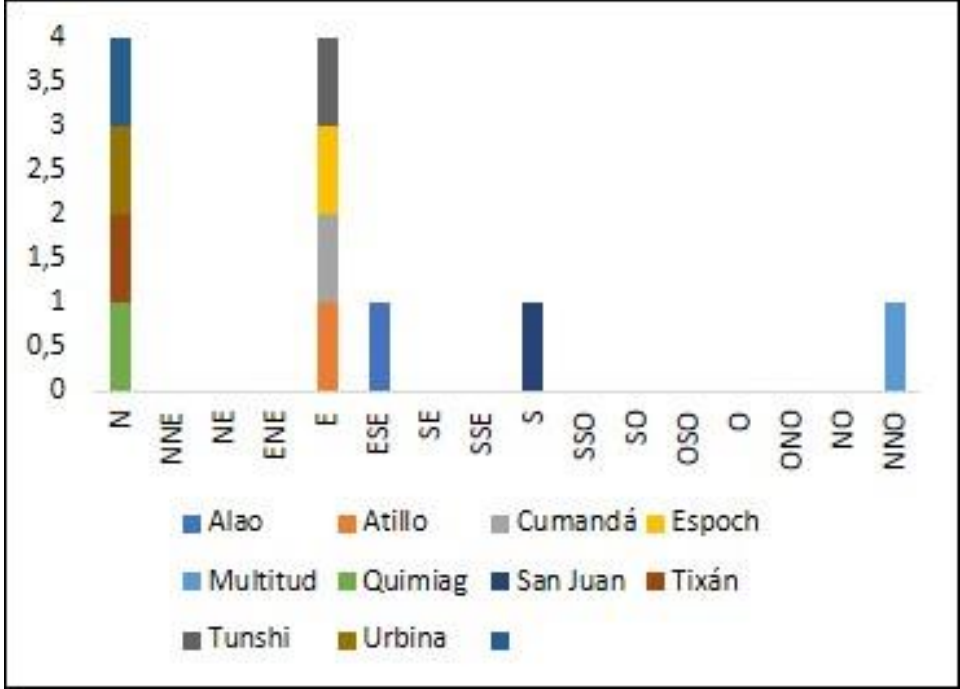

(d) 2017

Elaborado por: Diana Campoverde

En el 2015 (Figura 3 (b)) los vientos predominantes de Tunshi y Urbina se direccionan hacia el N; Atillo, Cumandá, Espoch, Multitud y Tixán hacia el E; Alao, Espoch y San Juan hacia el ESE; Multitud hacia S y Matus hacia el NNO. En el año 2016 (Figura 3 (c)) los vientos predominantes de Multitud, Tunshi y Urbina son hacia el N; de Quimiag hacia el NE; de Atillo, Espoch y Tixán hacia el E; de Alao y San Juan hacia el ESE; Atillo hacia el ONO y Matus hacia el NNO. En el año 2017 (Figura 3 (d)) los vientos predominantes de Multitud, San Juan, Tunshi y Urbina son hacia el N; de Atillo, Cumanda, Espoch y Tixán hacia el E; de Alao hacia el ESE; Quimiag hacia el S y Matus hacia el NNO. 
Visualizando las gráficas notamos que las estaciones de Cumandá, Multitud, Quimiag y San Juan son estaciones en las cuales sus direcciones de vientos predominantes no se mantienen constantes; Cumandá durante el año 2014 su viento predomina hacia el OSO, durante el 2015 hacia el Este, en el 2016 hacia el ONO y regresa hacia el E en el 2017; Multitud presenta direcciones de viento predominantes hacia el S durante los años 2014-2015 y cambia de dirección durante el 2016-2017, Quimiag presenta direcciones de viento predominantes hacia el NE durante el periodo de tiempo del 2014 al 2016 e inciertamente el año 2017 cambia de dirección hacia el S, San Juan presenta un caso similar a esta estación, durante los años 2014, 2015 y 2016 sus vientos predominan hacia el ESE y en el 2017 su dirección cambia con dirección hacia el N.

Geográficamente debido a su ubicación las estaciones de Cumandá y Multitud se encuentra ubicada cerca de la cuenca del rio Chanchan (SE), estas variaciones están influenciadas por su ubicación en relación al nivel del mar y la altitud de cada estación.

Variaciones diarias: Los registros de cada estación contienen información de mediciones de 1 hora entre cada registro durante las 24 horas del día, de los 4 años de estudio, cuyo cambio típico consiste en la velocidad del viento aumenta durante el día y desciende esa velocidad durante la medianoche y el amanecer, estas variaciones son importantes como una característica para evaluar ese potencial eólico.

Figura 4. Variación de la velocidad viento promedio por hora del viento durante el período 20142017 de cada una de las estaciones meteorológicas instaladas en la provincia de ChimborazoESPOCH.
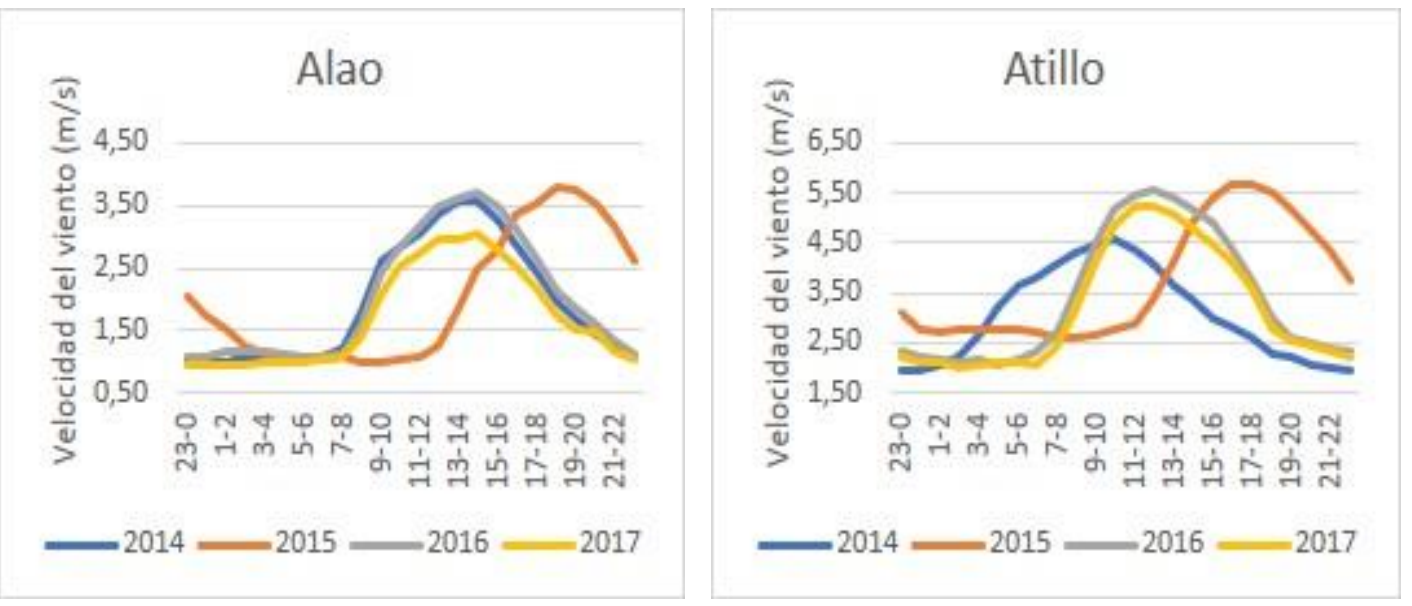

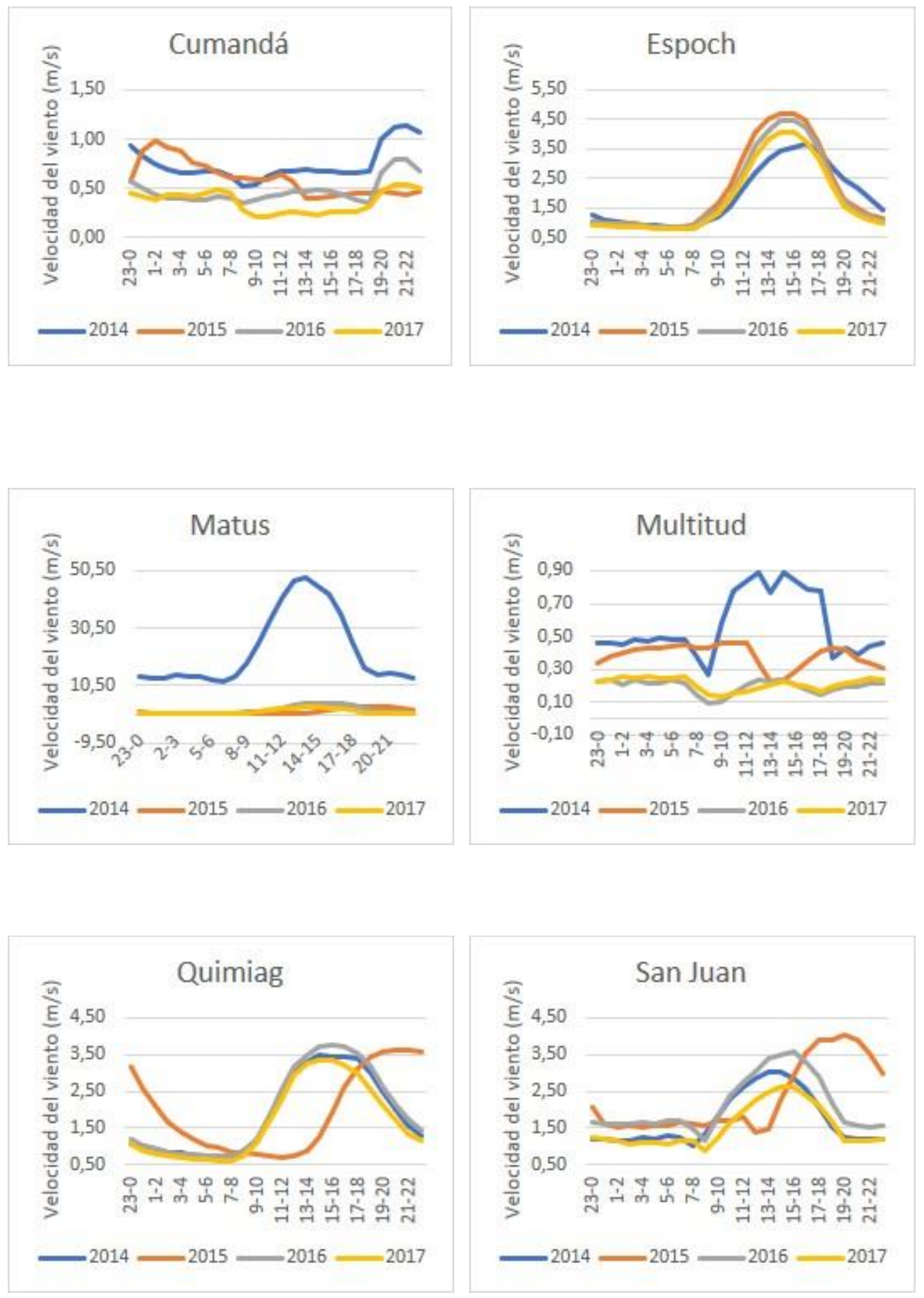
Vol. 3, $\mathrm{N}^{\circ} 1.1$, p. 389-407, febrero, 2020
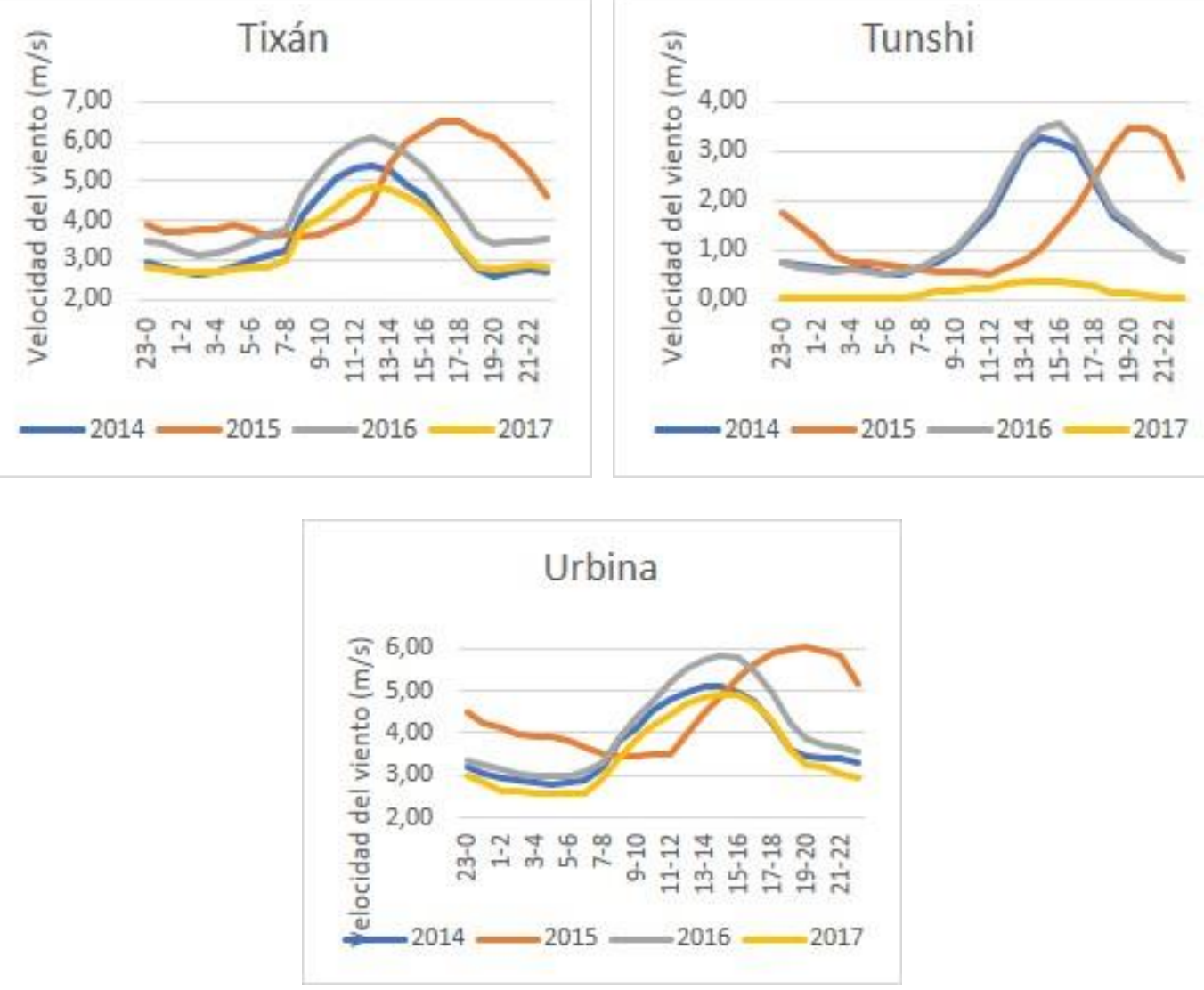

Elaborado por: Diana Campoverde.

La figura 4 presenta como característica más notable en las 11 estaciones que durante el día la velocidad del viento es superior al medio día, es decir desde las 12:00 hasta las 18:00, luego esta velocidad desciende hasta las 06:00, las estaciones de Cumandá y Multitud presentan un comportamiento especial, no se puede visualizar un patrón común en los 4 años de estudio.

Se construyeron rosas de viento (Figura 5) de cada una de las estaciones para los 4 años en estudio, que asocian tanto la distribución de la velocidad como de la dirección. Las rosas de viento muestran las direcciones predominantes y las velocidades en 5 clases de $3 \mathrm{~m} / \mathrm{s}$ de rango por colores: el color verde para velocidades mayores a $9 \mathrm{~m} / \mathrm{s}$, el color azul para velocidades entre 7 y $9 \mathrm{~m} / \mathrm{s}$, el color rojo para velocidades entre 5 y $7 \mathrm{~m} / \mathrm{s}$, el amarillo para velocidades de 3 a $5 \mathrm{~m} / \mathrm{s}$ y el color gris claro para velocidades de 0 a $3 \mathrm{~m} / \mathrm{s}$.

Finalmente se exportó las rosas de viento a Google Earth de las 11 estaciones meteorológicas con el fin de visualizar y asociar las variables estudiadas con su posición geográfica (Figura 6).

Los resultados mensuales de velocidad y dirección de viento dieron lugar a 528 rosas de viento (12 meses, 11 estaciones y 4 años), motivo por el cual no se las presenta en este paper, sin embargo, en la discusión, se hace hincapié en las características encontradas en su comportamiento. 


\section{EDigital}

ISSN: 2600-5859

Vol. 3, N¹.1, p. 389-407, febrero, 2020

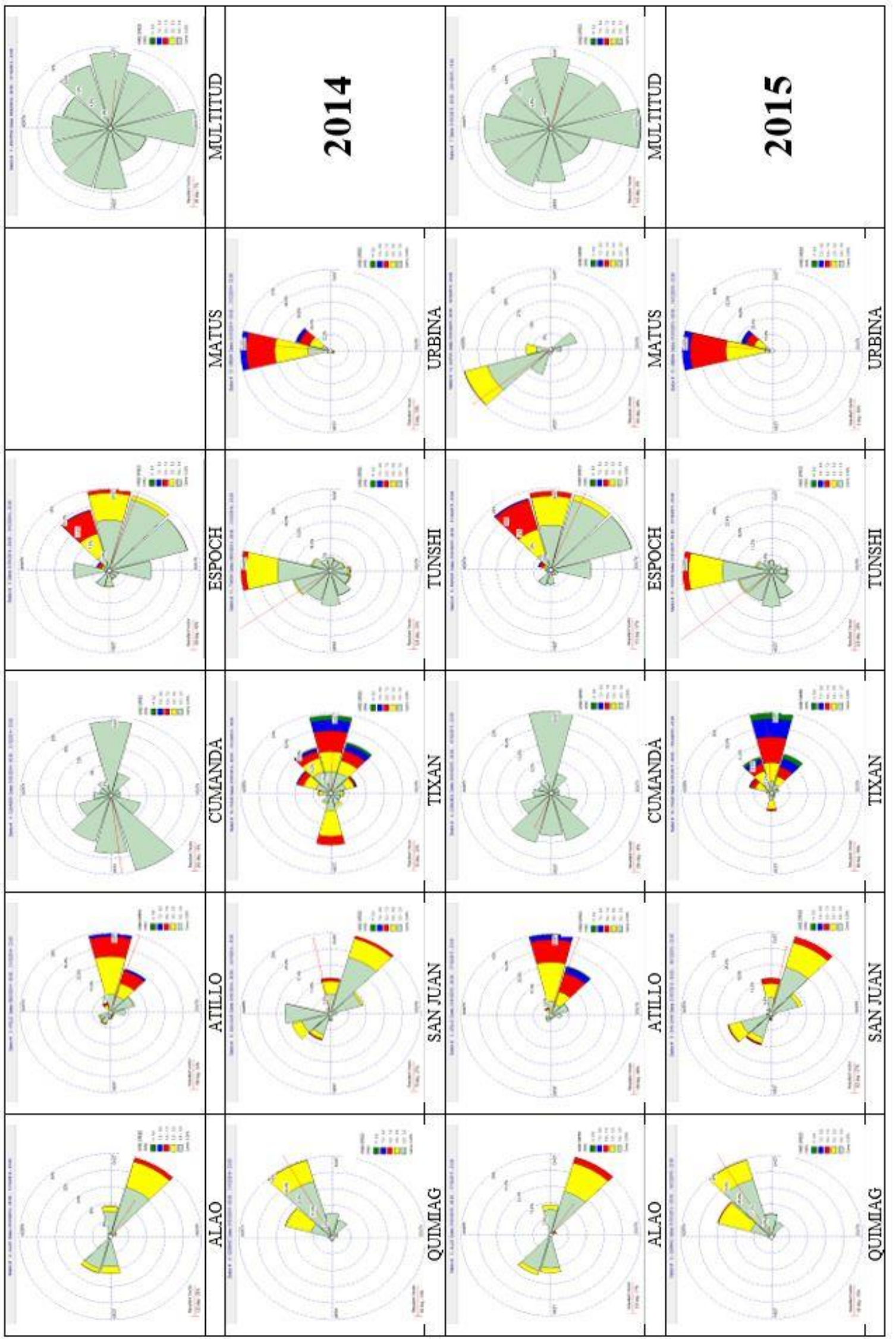

Creatividad, Diseño e Innovación 


\section{(DDigital}

ISSN: 2600-5859

Vol. 3, $\mathrm{N}^{\circ} 1.1$, p. 389-407, febrero, 2020

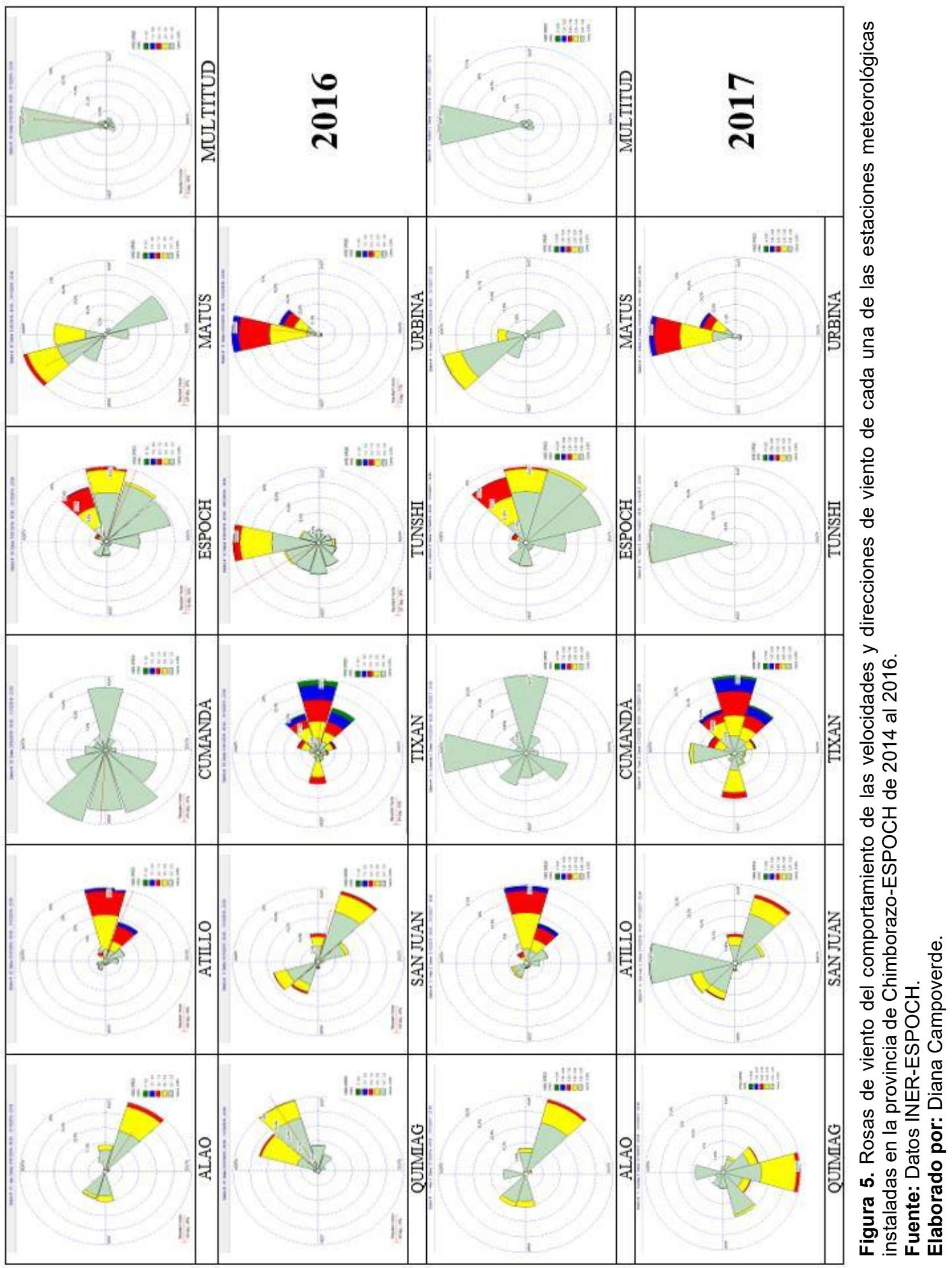

_Creatividad, Diseño e Innovación 


\section{EDigital}

ISSN: 2600-5859

Vol. 3, $\mathrm{N}^{\circ} 1.1$, p. 389-407, febrero, 2020

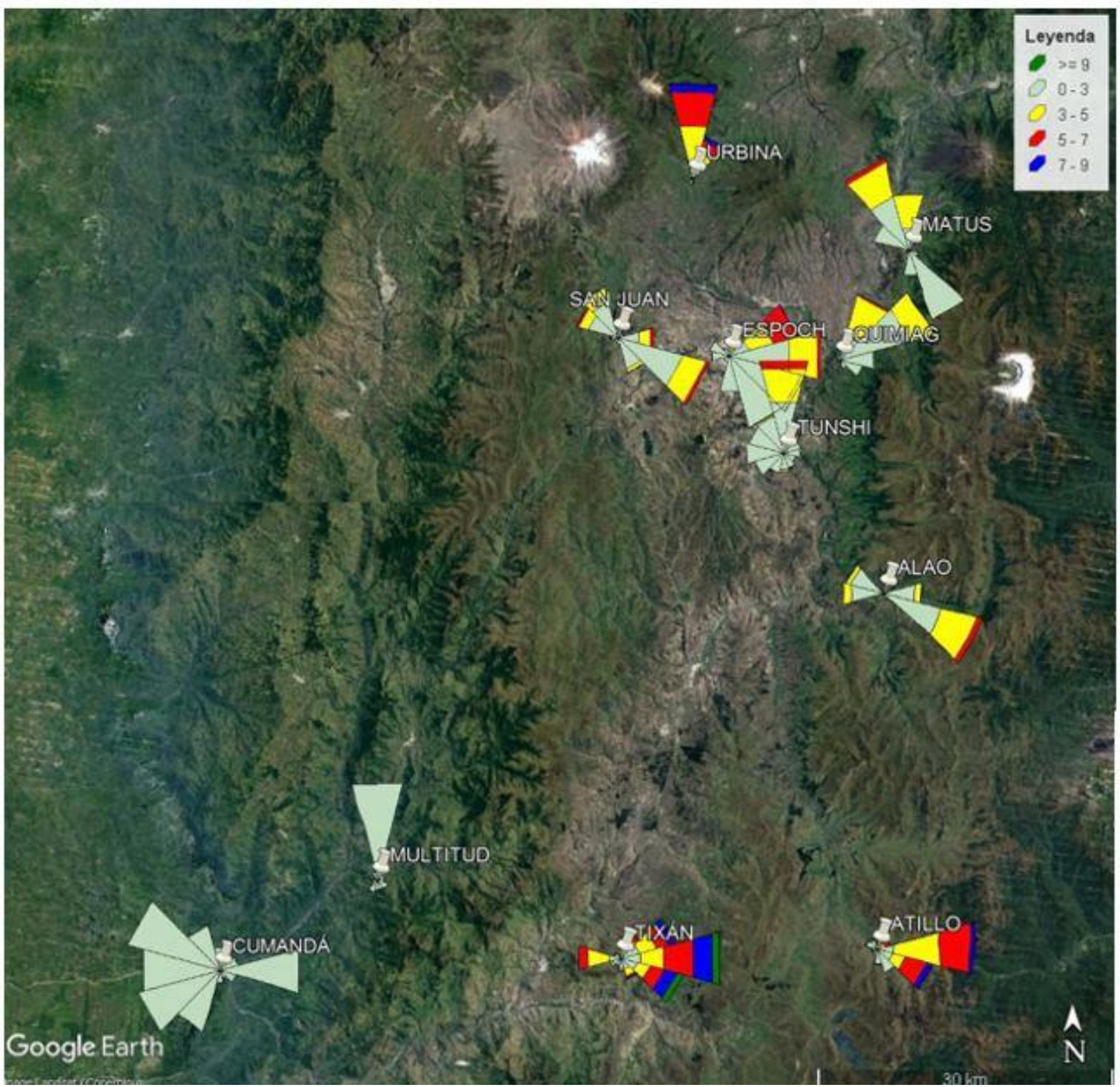

Figura 6. Rosas de viento de las 11 estaciones meteorológicas instaladas en la provincia de ChimborazoESPOCH de 2014 al 2017.

Elaborado por: Diana Campoverde

\section{Conclusiones.}

- El análisis correlacional de las direcciones de viento con la temperatura del aire, radiación solar global, humedad y precipitación no mostró influencia de dichas variables en su comportamiento, en la actualidad una de las problemáticas es el desarrollo de fuentes energéticas a partir de recursos eólicos, esto ha motivado a realizar estudios de caracterización que permitan evidenciar el alto potencial de energía eólica por explorar y utilizar, que permitan seleccionar de manera decisiva la aplicación de este recurso.

- El análisis anual y mensual identificó estaciones con direcciones de viento predominantes estables en: Alao hacia el SE, Atillo hacia el E, Espoch hacia el E, Matus hacia el NNO, 
Tixán hacia el E, Tunshi y Urbina hacia el N. Notándose un comportamiento diferente en Cumandá al estar ubicada en la cuenca del rio Chanchan (SE) y el río Tixan (NE) provocando cambios bruscos en la dirección de viento, en aproximadamente un ángulo de 135 grados. Las direcciones de viento predominantes durante los años de estudio se clasificaron en dos grupos: de enero a junio los vientos son predominantes hacia el $\mathrm{E}$ en los cuatro años de estudio y de julio a diciembre presentan vientos predominantes con dirección hacia el SO en los años 2015 y 2016, mientras que en los años 2014 y 2017 presentó vientos predominantes hacia el NO, característica que no está estrechamente asociado con las época cálida y lluviosa de la región costa (de junio a abril); Multitud está influenciada por las microcuencas de los ríos Maguazo (NE) y Chimbo (SO) generando direcciones de viento con menor estabilidad de enero a junio con una dirección de $\mathrm{N}$ a $\mathrm{S}$ $(2014,2015,2016$ y 2017) y mayor estabilidad de julio a diciembre con vientos predominantes al E (2014 y 2015), al SO, S y SE (2016) y al N (2017). Ligeramente se detectaron comportamientos variantes aproximadamente cada 4 meses en ángulos de 45 hasta 180 grados, no se identificó comportamiento asociado a las épocas secas y lluviosas de la sierra y costa; Tixán se encuentra en el centro de las microcuencas de los ríos Zula (SE) y Sibambe (NE) provocando vientos predominantes con dirección estable hacia el E; Espoch se halla dentro de la microcuenca del río Chibunga, la dirección predominante de viento varía de N, NE, E, SE y S durante todo el año.

- Los resultados obtenidos a partir de este estudio identificaron que las estaciones con más altas velocidades de viento son Tixán y Urbina, ya que presentan registros de velocidades entre $\operatorname{los} 3$ y 4,0 m/s del 2014 al 2017, causando la posibilidad de aplicar modelos de generación de energía mediante molinos de viento previo a un estudio de rentabilidad.

- El comportamiento de las velocidades y direcciones de viento no presentan patrones comunes de comportamiento, es decir, la provincia de Chimborazo presenta múltiples ecosistemas debido a la diversidad de comportamientos de dichas variables, puesto que van en función de la ubicación geográfica de la zona.

- El software Wrtplot no destaca las características atípicas de la velocidad y dirección de viento, los resultados no mostraron diferencias significativas entre el análisis de estas variables con y sin un proceso previo de limpieza de datos, por lo que es una ventaja, ya que al no poseer suficiente conocimiento estadístico para realizar dicho análisis no imposibilita la detección de vientos predominantes. Otra ventaja del software, se destaca al momento de realizar la imputación; Wrtplot realiza el relleno automático de datos faltantes sin ningún problema.

\section{Referencias bibliográficas.}

Atkinson, D., \& Russell, F. L. (1992). Procedures for Substituting Values for Missing NWS Meteorological Data for Use in Regulatory Air Dispersion Models. 
Bichet, A., Wild, M., Folini, D., \& Schär, C. (2012). Causes for decadal variations of wind speed over land: Sensitivity studies with a global climate model. Geophysical Research Letters, 39(11).

Bultó, P. L. O., Rodríguez, A. E. P., Valencia, A. R., Carreras, A. P., Cangas, J. R., \& Estela, L. B. L. (2008). La variabilidad y el cambio climático en Cuba: potenciales impactos en la salud humana. Revista Cubana de Salud Pública, 34(1).

Carta, J. A., Ramírez, P., \& Velázquez, S. (2009). A review of wind speed probability distributions used in wind energy analysis. Case studies in the Canary Islands. Renewable and Sustainable Energy Reviews, 13(5), 933-955.

Cellura M., Cirrincioneb G., \& Miraoui, A. (2008). Wind speed spatial estimation for energy planning in Sicily: Introduction and statistical analysis. Renewable Energy, 33(6), 12371250.

Ciampoli, M., Petrini, F., \& Augusti, G. (2011). Performance-based wind engineering: towards a general procedure. Structural Safety, 33(6), 367-378.

Cruz, C., Sebrango C., Hernánez, M., Pina, C., Marquetti, M., \& Sánchez, L. (2010) Comportamiento estacional y temporal de Aedes aegypti (Diptera: Culicidae) en Sancti Spíritus, 1999-2007. Revista Cubana de Salud Pública, 62(1), 1-10.

Cúneo, L. M., Cerne, S. B., \& Llano, M. P. (2018). Descripción preliminar de la velocidad y dirección del viento medio mensual en Trelew. Centro Argentino de Meteorólogos; Meteorológica; 44; 1; 4-2018; 66-80

De la Cruz Buelvas, J., Valencia Ochoa, G., \& Vanegas Chamorro, M. (2018). Statistical study of wind speed and direction in the departments of Atlántico and Bolivar in Colombia. Ingeniare. Revista chilena de ingeniería, 26(2), 319-328.

Gökçek, M., Bayülken, A., \& Bekdemir, S. (2007). Investigation of wind characteristics and wind energy potential in Kirklareli, Turkey. Renewable Energy, 32(10), 1739-1752.

Haro, S., Zúñiga, L., Vera, L., Meneses, A., \& Escudero, A. (2018). Métodos de clasificación en minería de datos meteorológicos.

Hennessey Jr, J. P. (1977). Some aspects of wind power statistics. Journal of applied meteorology, 16(2), 119-128.

Köse, R. (2004). An evaluation of wind energy potential as a power generation source in Kütahya, Turkey. Energy conversion and management, 45(11-12), 1631-1641.

Natalini, B., \& Natalini, M. (2013). Revisión de velocidades de viento extremas en tres localidades del sur de Argentina. Rev. Int. de Desastres Naturales, Accidentes e Infraestructura Civil, 13(2), 235-248.

Navarro, M. (1990). Frecuencia e intensidad del viento en Zaragoza. Geographicalia, 27, 63-75.

Olioso, A., Ortega, S., Valdés, H., \& Antonioletti, R. (2005) Estimación de la Evapotranspiración de Tomate Usando el Modelo Interacción Suelo - Vegetación - Atmósfera (ISBA). Agricultura Técnica, 65(3), 284-294. 
Ortíz, P., Pérez, A., Rivero, A., Pérez, A., Cangas, J., \& Bartolomé, L. (2008). La variabilidad y el cambio climático en Cuba: potenciales impactos en la salud humana. Revista Cubana de Salud Pública, 34(1).

Ovando, G., Bocco, M., \& Sayago, S. Redes neuronales para modelar predicción de heladas. Agricultura Técnica, 2005, 65(1), 65-73.

Peña, D. (2013). Análisis de datos multivariantes. McGraw-Hill España. Pérez, N., Salomón, H. \& Marcatoma, J. (2020). Análisis del cambio climático en un ecosistema alto andino, Riobamba-Ecuador. Rev. Perfiles, 1(23), 4-11.

Pizarro, O., Hormazabal, S., González, A., \& Yañez, E. (1994). Variabilidad del viento, nivel del mar y temperatura en la costa norte de Chile. Investigaciones marinas, 22, 85-101.

Quiñonez Choquecota, J., Huanca Callata, E., \& Holguino Huarza, A. (2019). Caracterización del recurso eólico en la ciudad de Juliaca. Revista de Investigaciones Altoandinas, 21(1), 5768.

Rivera, S., \& Escudero, A. (2017). Interpolación de datos faltantes mediante spline cúbico y mínimos cuadrados. Rev. Perfiles, 1(17), 63-66.

Tamura, A. (2015). Advanced structural wind engineering. A. Kareem (Ed.). Springer Verlag, Japan.

Ucar, A. \& Balo, F. (2008). A Seasonal Analysis of Wind Turbine Characteristics and Wind Power Potential in Manisa, Turkey. International Journal of green energy, 5, 466-479.

Valencia, G., Vanegas, M., \& Polo, J. (2016). Análisis Estadístico de Velocidad y Dirección del Viento en la Región Caribe Colombiana con Énfasis en la Guajira. Sello Editorial Universidad del Atlántico, 1, 28-53.

Watson, S. (2014). Quantifying the variability of wind energy. Wiley Interdisciplinary Reviews: Energy and Environment, 3(4), 330-342..

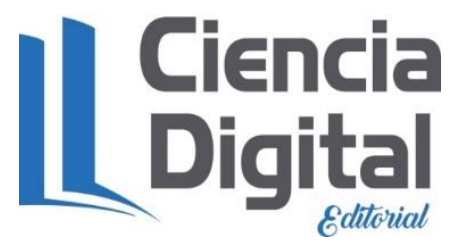




\section{PARA CITAR EL ARTÍCULO INDEXADO.}

Campoverde Santos, D. K., Escudero Villa, A. I., \& Haro Rivera, S. M. (2020). Caracterización de la velocidad y dirección de viento en la provincia de Chimborazo. ConcienciaDigital, 3(1.1), 389407. https://doi.org/10.33262/concienciadigital.v3i1.1.1156

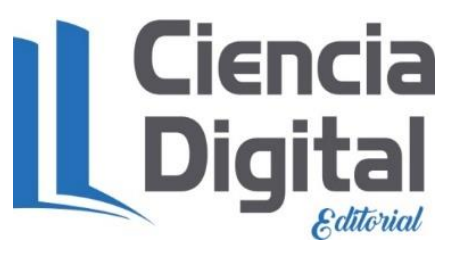

El artículo que se publica es de exclusiva responsabilidad de los autores y no necesariamente reflejan el pensamiento de la Revista Conciencia Digital.

El artículo queda en propiedad de la revista y, por tanto, su publicación parcial y/o total en otro medio tiene que ser autorizado por el director de la Revista Conciencia Digital.

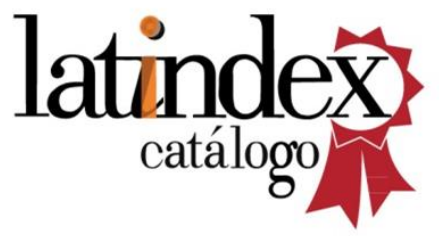

\section{Conciencia}

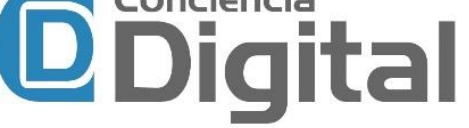

